\title{
Immersive Design Engineering
}

\author{
Bjorn Sommer ${ }^{1, \$}$, Chang Hee Lee ${ }^{1}$, Nat Martin ${ }^{1}$, Vanna Savina Torrisi ${ }^{1}$ \\ ${ }^{1}$ Innovation Design Engineering, Royal College of Art, London, UK \\ \$Corresponding author: bjoern@CELLmicrocosmos.org
}

\begin{abstract}
Design Engineering is an innovative field that usually combines a number of disciplines, such as material science, mechanics, electronics, and/or biochemistry, etc. New immersive technologies, such as Virtual Reality (VR) and Augmented Reality (AR), are currently in the process of being widely adapted in various engineering fields. It is a proven fact that the modeling of spatial structures is supported by immersive exploration. But the field of Design Engineering reaches beyond standard engineering tasks.

With this review paper we want to achieve the following: define the term "Immersive Design Engineering", discuss a number of recent immersive technologies in this context, and provide an inspiring overview of work that belongs to, or is related to the field of Immersive Design Engineering. Finally, the paper concludes with definitions of research questions as well as a number of suggestions for future developments.
\end{abstract}

\section{Introduction}

Design Engineering is an innovative area that stays at the intersection of fields, combining a number of diverse disciplines, such as architecture, material science, biochemistry, mechanics, electronics, physics textiles etc. But besides disciplines that are more closely related to the process of making, there are also those devoted towards economics [1].

New immersive technologies related to, e.g., Virtual Reality $(V R)$ and Augmented Reality (AR), are currently in the process of being widely adapted in various engineering fields. It is a proven fact that the modeling of spatial structures is supported by immersive exploration [2, 3]. But the field of Design Engineering reaches beyond standard engineering tasks. Robinson et al. define for example 49 competencies relevant in the Design Engineering field [1]. And although design engineers spend a decent amount of time doing solo work - especially during technical tasks - they are in some areas highly dependent on collaborative work, especially in terms of decision making and information understanding [4]

This paper is structured the following way:

- First, we will introduce immersive technologies related to VR and AR.

- Second, we make a first attempt to establish a definition for the field of "Immersive Design Engineering".

- Third, we are introducing a number of research projects that belong to, or are related to the field of "Immersive Design Engineering". Please note that a fully comprehensive review is out of the scope of this paper.

- This is followed by a quick overview of related commercial projects whose roots go back to student projects or research project. This also includes a concrete example of a student semester project that recently became a startup.
- Then, in the Results and Conclusion chapter we will sum up what we learnt from the overview of projects.

- And finally, in the Outlook, we will present a few new ideas that might impact future Immersive Design Engineering work.

\section{The Base: VR and AR Technologies}

Virtual Reality (VR) and Augmented Reality (AR) technologies are currently in the process of being widely adapted in various engineering fields. VR technologies - such as Oculus Rift ${ }^{\mathrm{TM}}$ and HTC VIVE ${ }^{\mathrm{TM}}$ - have high potential to survive the hype of VR as they are quite affordable and widely adapted in a number of application fields. Since 2019 the extension of the product portfolio shows a number of promising new developments, enabling also more mobile solutions. It is widely accepted that the exploration of spatial objects, such as cars, houses and medical scans, benefit from VR technologies, as it improves the understanding of spatial structures and compositions [2] 3].

Although the COVID-19 pandemic lead to a decrease in shipments during the first half of 2020 - especially because of disrupted supply chains - the long-term outlook is positive, according to IDC [5]. The lock-down situation lead to a temporary working-from-home society and is expected to drastically increase the interest in VR technologies.

As an alternative to VR headsets, semi-immersive 3Dstereoscopic displays (such as Power Walls) were often seen in engineering-related scenarios in the past. For research and development, CAVEs were an especially promising virtual environment [6, 7]. Many of these screen-based VR technologies barely survived the decline of the commercial 3D-TV market [8].

But it would be wrong to expect that the same destiny will apply to all VR technologies on the market. Whereas full immersive Head-mounted displays (HMDs) like Oculus Rift and HTC VIVE might not meet the expectations of all professional audiences, products like the Varjo XR-1 provide already a convincing alternative, in terms of resolution (3000 PPI), comfort (up to $90 \mathrm{~Hz}$ refresh rate) and tracking, and provide stereoscopic seethrough enabling AR applications (12 MP at $90 \mathrm{~Hz}$ ) [9, 10, 11].

Due to the decline of 3D TVs, it became harder to find 3D displays like Fishtank displays. However, as an appropriate alternative the zSpace display and laptop provide a passive stereoscopic display [12]. Although this display requires glasses, it comes with the big advantage of integrated high-resolution head tracking. Moreover, it comes with a 3D stylus pen.

Years ago Planar provided the popular StereoMirror technology, which made use of two LCD displays and merged the two images on a polarized mirror between both displays - polarized glasses were required. Although Planar does not manufac- 
ture these displays anymore, new providers like Schneider Digital created the 3D PluraView enabling up to $4 \mathrm{~K}$ resolution for each eye [13].

Although there have been auto-stereoscopic displays around for a long time, most of them were just prototypes or not really unusable. Nowadays, the Looking Glass Display is an example of a very user-friendly product and might be the currently the most widely-adopted holographic display [14]. A wider range of autostereoscopic flat displays is provided by Alioscopy $($ - from Full HD to $4 \mathrm{~K}$ and different formats [15]. Alioscopy is indeed an example of a successful S3D technology company that has been in the market since 1986.

Another very popular AR/Mixed Reality device is the Microsoft Hololens which was used to build many prototype applications in the engineering field [16]. However, the limited interaction capabilities and field of view of the first HoloLens version prevented that it being widely adopted by the industry. This might change with the HoloLens 2 as the hand interaction has been drastically improved.

One of the milestones in VR was the development of the CAVE [6]. These multi-display and multi-side environments (e.g. top, front, left and right side) can be only afforded by large companies, such as car manufactures or universities. The CAVE2 is an alternative version of the CAVE, which consists of multiple 3D displays [7]. Mechdyne is the company which provides many of these technologies and has many patents in this area [17]. Last but not least, for tracking in complex environments, often VICON tracking technology is used [18].

Obviously, these mostly hardware technologies require an engine to drive them. In the recent years, a number of VR development engines have been established. According to Checa et al. who evaluated a number of virtual reality serious games in the context of learning and training, the most-widely used platforms in gaming industry are Unity and the Unreal Engine, whereasWorldviz and the Open Source VR platform Ogre3D were mainly used for older projects [19, 20, 21, 22, 23]. But in the Education and Training sector, Unity dominates, especially as it it has been available at a low cost for many years. However, a strong growth for the Unreal Engine is expected, as it converted to free software and provides better, photorealistic rendering capabilities.

\section{Immersive Design Engineering}

Nowadays, many professions steadily grow in terms of complexity due to their interdisciplinary nature. This applies especially to the field of "Design Engineering". While already a complex field with many specializations, this field is also under steady development and transformation.

Before looking into several approaches that could support Design Engineering through immersive technologies, we would like to propose a definition for this field.

\section{"Design Engineering" is defined by the McGraw-Hill Dictionary of Scientific \& Technical Terms as: \\ "A branch of engineering concerned with the creation of sys- tems, devices, and processes useful to and sought by society." [24]}

As previously mentioned, Design Engineering is a complex field and this definition might not be able to capture all nuances, but we think that this is an appropriate general definition.
"Immersive Design Engineering" we propose to define as:

"A branch of engineering concerned with the creation of systems, devices, and processes useful to and sought by society immersing the actor by making use of new immersion-supporting technologies. This definition includes, but is not limited to software systems, hardware devices, as well as production processes. Immersive Design Engineering (IDE) applies to the creation process of related hardware devices, as well as their usage in associated systems and processes. Therefore, all products of IDE might be temporarily or permanently components of a circular process. To achieve an appropriate level of the actor's immersion, a task-pragmatic selection of involved senses has to be made."

We previously discussed a number of VR and AR technologies. Following the definition above, is, i.e., an HMD already an Immersive Design Engineering project?

From a theoretic perspective, let us assume, a specific HMD is developed without actually using the technology. The optics are developed and implemented based on calculations, the HMD's body is designed with general 3D modeling software while using a standard 2D monitor and then the body is 3D-printed, sensors are integrated, and the according software and tracking system is developed based on specifications without actually using the HMD in the process. The final HMD product is plugged into a computer system and it works like expected. In this case, this particular HMD is not an Immersive Design Engineering project, but it can be used from now on to work in the context of Immersive Design Engineering projects. However, as the development of HMDs will always be accompanied by continuous testing and evaluation of a number of fragments and prototypes involving substantial immersive experiences, the development of VR technologies can nearly always be seen as an Immersive Design Engineering project.

"Immersion" is of course not a novel term in the design domain. Lidwell, Holden and Butler defined Immersion as "A state of mental focus so intense that awareness of the 'real' world is lost, generally resulting in a feeling of joy and satisfaction" [25]. They also note that the term is complex and controversial and that "perceptual immersion is more difficult to sustain for long periods of time and is, therefore, usable only for relatively brief experiences. Optimal immersive experiences involve both rich sensory experiences and rich cognitive engagement" [25].

Furthermore, they characterized Immersion by a number of elements [26]. In the context of Design Engineering, just a selection should be mentioned: challenges that can be overcome, contexts where a person can focus without significant distraction, clearly defined goals, immediate feedback with regards to actions and overall performance, a modified sense of time, as well as a feeling of control over actions, activities, and the environment [25].

According to Lidwell, Holden and Butler, already one of these elements might be sufficient to create Immersion. Naturally, there are various degrees of Immersion. As previously mentioned, in the context of VR technologies, an HMD is usually characterized as fully immersive, whereas a $3 \mathrm{D}$ screen is seen as semiimmersive. It is obvious that in this context the visual cue has the highest impact and one could argue that an experience cannot be fully immersive in case, e.g., audio support is missing. But even 
the full audiovisual experience would still exclude the remaining senses.

The ultimate Immersion would require the involvement of all senses. Usually, recent VR applications support the visual, audio and haptic sense (the latter mostly only in a very basic way using vibration), whereas other senses are neglected. However, there long exists research in this area. Just to give one examples: Years ago, Hülsmann et al. simulated wind and warmth in VR inside a CAVE by using a very affordable setup consisting of fans and infrared lamps [27].

But to come back to the selection of terms characterizing immersion: it was mentioned, that a selection might be sufficient to deliver immersion. Especially in terms of Design Engineering, it will usually not be required to support all senses, as Design Engineering is purpose driven and focus on a highly specific task, the support of all senses is not required.

\section{Related Projects}

Here, we want to explore perspectives of Immersive Design Engineering, by discussing potential application scenarios and illustrating examples. In terms of Design Engineering, the amount of available examples is still quite limited. However, we want to present here a number of examples that are Design Engineering projects, or which can inspire their future development.

\section{Virtual Cities, Landscapes and Cultural Heritage Auto-stereoscopic Display Design}

Whereas a few previously-mentioned VR technologies are already able to render objects in a resolution that is appropriate for most application cases, autostereoscopic displays usually come with the disadvantage of a lowresolution for each eye. Barre et al. present a new autostereoscopic design for lenticular lens displays in conjunction with an optimized algorithm to display the stereoscopic images [28]. The difference of the discussed approach to common autostereoscopic two-view-designs with lenticulars is that it allows more distance between the image splitter and display panel - the resulting low magnification factor reduces distance errors from relative panel/splitter movements. The system was integrated and tested in a production chain for digitalization and presentation of scanned 3D objects from the Fraunhofer cultural heritage project. Using web technology with a $\mathrm{x} 3 \mathrm{dom}$ renderer, autostereoscopic rendering as well as interaction with the objects was enabled by providing hand and head tracking.

\section{Simulation of a Historic City}

The sensiLab at Monash University is a very experimental workplace that uses VR technologies in the context of speculative design [29]. The Angkor Wat Virtual Environment developed at the sensiLab is an excellent example of how VR can be used to explore ancient cities and architecture to elaborate different theories of their internal functionality using crowd simulation. In this way researchers can analyze potential routes a pilgrim might have taken when visiting the temple of Angkor Wat [30]. Similar approaches could be used in architectural design to evaluate the feasibility of buildings during the design process and test different scenarios, such as disaster management, way finding, shortest path analysis, etc.

\section{Generation of Virtual Cities optimized for Virtual Envi- ronments}

Another interesting method is to generate virtual environments optimized for immersive environments [31]. During the generation of virtual cities, stereoscopic parameters are used to automatically place objects inside the stereoscopic camera frustum avoiding to reach an excessive parallax on screen and reducing/avoiding window violations. In the context of this work, a plugin for the Open Source Software Blender was created to generate the procedural cities. Using the standard parameter (with $s f f=1.0$ ), the parallax on screen of the models on the far background will be approximately equal to the maximum parallax on screen achievable before divergence occurs. Figure 1 shows a city created with $s f f=0.5$. Keyword: Architecture, City Planning, Paper: Application

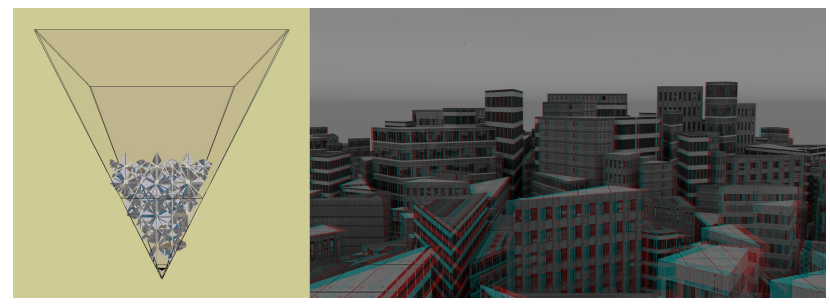

Figure 1. A Blender-based procedural engine fills the stereoscopic camera frustum with geometry according to a user-provided value. Images on the right can be viewed with anaglyph red-cyan glasses. (Courtesy of (C)2016 Davide Gadia. All Rights Reserved.)

\section{Cloud-based Exploration of Sensor Data}

The prototype of the Urban Insight Cloud Engine (UICE) was developed to visualize and explore sensor data using a cloudbased system. A prototype was implemented to explore the city of Newcastle-upon-Tyne using web browsers as well as S3D display setups [32]. This paper also lists a number of design decision for large-scale immersive display environments are listed, which are: Ease of use, Easy to maintain, Standard aspect ratio, Bright, high resolution, high frame rate, 3D, Simple interaction devices. A number of different larger S3D display setups are discussed and compared that are partly used to support decision finding in groups.

\section{Sensor Data Visualization}

Baltabayev et al. discussed how two simple mobile VR approaches can be used to explore 3D terrain in combination with sensor data with very simple smart phone setups [33]. For this purpose, two Android apps were developed for the data recording task, and a standard web browser was used in combination with WebVR to visualize the virtual environments. A simple terrain visualization was created and sensor data was associated with the underlying coordinates. The coordinates were either recorded based on GPS sensors (low spatial resolution, Figure 2) or by trilateration between WiFi hotspots (high spatial resolution). This work shows that it is possible with very simple mobile solutions to record and visualize sensor data. 


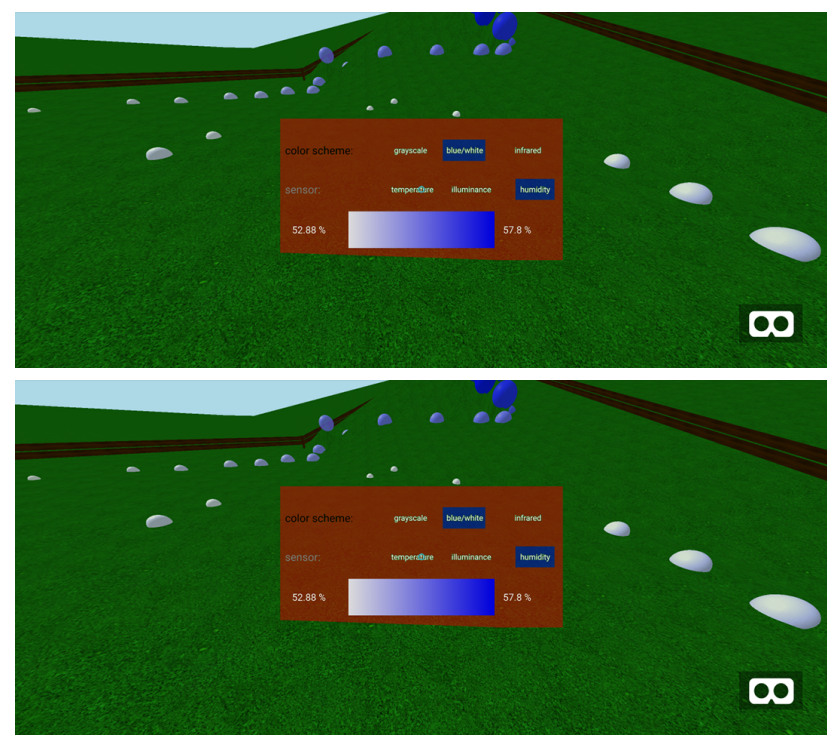

Figure 2. $3 D$ world shown on a smart phone using a web browser. The color scheme is encoding humidity and the surface of the virtual environment was created based on GPS data. The data selection dialog is shown together with the color scale.

\section{Vehicle Simulation and Remote Control Vehicle Simulation and Exploration}

Miranda et al. were using a vehicle simulator with motion capability combined with a CAVE to elaborate physiological driver data [34]. More precisely, data of breathing, galvanic skin resistance (GSR) and pressure on the hand of the participant were measured with sensors. A number of interesting observations were collected, e.g. respiration signals are good indicators for characterizing stress variation, or that the simulator can identify changes in user behavior through the physiological signs of breathing, pressure and GSR and its analysis using the average frequency throughout the simulation period. In this way, these virtual simulation environments are adequate alternatives for R\&D in the car industry as long as a car prototype was not built.

\section{Improving Remote Activities through Stereoscopic Vision} Boonsuk elaborated the use of S3D visualization to help improve user performance in remote activities. Experiments were conducted using a Oculus Rift DK2 with separate left/right video streams with 12 participants. The videos were fed from two Raspberry PI cameras attached to a remote car (Figure 3). Participants using S3D visualization had estimated significantly better performance in relative distances between remote car and a wall. These results could be extended to help designers develop teleoperating viewing system for devices like remote control cars, drones, and tele-guided robots [35].

\section{Navigation}

\section{Distance Measurement and Estimation}

Deas et al. in line with previous work showed that stereoscopic vision is especially useful to estimate the relative altitude in relation to a terrain at low altitudes $(0-5 \mathrm{ft})$. In contrast, binocular vision provides very weak input to absolute altitude estimates at high altitudes (10-100ft) [36]. During their evaluation of absolute

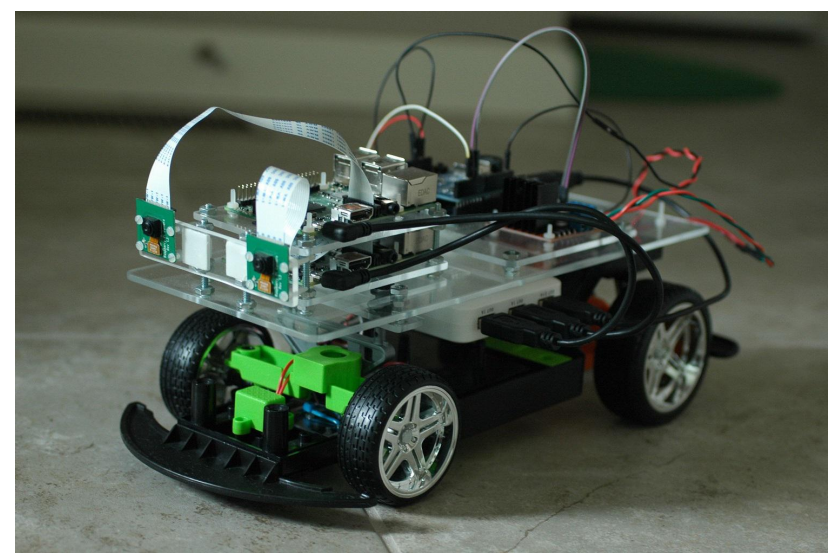

Figure 3. This remote control car is equipped with two cameras for $S 3 D$ real-time video transfer. Participants of the user experiment had to stop the car in front of a wall using either monoscopic or stereoscopic vision. The latter outperformed the monoscopic approach. (Courtesy of (C)2015 Wutthigrai Boonsuk. All Rights Reserved.)

and relative distance measurements, real world scenes were compared using natural as well as simulated S3D imagery. For this purpose, a S3D monitor was installed which showed a) the S3D image/scene, and b) an extension of a real-world tube into the virtual world (representing a helicopter skid) that was rendered on top of a. As the head position as well as the distance between S3D monitor and participant were fixed during the experiment, the skid provided a convincing spatial reference when measuring distances.

\section{Semantics for Integrated Pipeline for Immersive Environ- ments}

Trellet et al. discussed an integrated pipeline designed for immersive environments, promoting direct interactions on semanticallylinked 2D and 3D heterogeneous data presented in a common working place [37]. The combination of data is facilitated by 1) making use of pre-existing/inferred links present in our hierarchical concepts' definition, 2) enrichment by using task-dependent and adaptive analyzes proposed to the user, and (3) interactively presented in a unique working environment to be explored.

\section{Avoiding cybersickness}

A relevant issue of daily work inside virtual environments is cybersickness. In case a joystick is used to trigger rotations, fast motions often lead to cybersickness as the user fully visually perceives the motion in the virtual world. This is also a reason why teleportation is frequently used in virtual environments instead of moving fluently forward using controllers. To reduce cybersickness by optimizing rotational motions, for example Kemeny et al. developed a new navigation technique called "Head Lock" [38]. This method is based on thresholds obtained by experiments which are used to keep fixed visual outside-world references. Closing a single eye persistently will bind the virtual environment to the user's head. When the rotation is finished, the user opens this eye again and the locking mechanism is disabled. 


\section{Hybrid-dimensional visualization and navigation}

The paper [39] discusses hybrid-dimensional visualization of biomedical and functional structures. It discusses new ways of interaction with a stereoscopic monitor - the zSpace - by using head-tracking for navigation. The head-tracking can be used to navigate around the car model. Using the zSpace stylus pen (Figure 5 Top), the user can interact with objects in 3D space. The $3 \mathrm{D}$ visualization is supplemented by a simple $2 \mathrm{D}$ map showing the different components of the car as technical terms (Figure 4). Clicking on one of these terms directly navigates the $3 \mathrm{D}$ view to the corresponding perspective and focuses the object of interest here, the left-front wheel.

\section{Stereoscopic Space Map}

The Stereoscopic Space Map is a system created for group presentations in a CAVE2 environment consisting of 80 stereoscopic monitors, communicating with a central computer connected to a zSpace monitor (Figure 5Top). This system would enable the immersive exploration of large virtual environments with a number of experts and engineers by providing a $330^{\circ}$ panoramic view [40]. The central navigator is able to first check potential perspectives on the zSpace (Figure 5 center) - here, inside an animal cell - and then smoothly navigate the complete virtual environment (shown in the CAVE2) to this specific location (Figure 5 Bottom). This system tries to tackle the problem that the $\mathrm{S} 3 \mathrm{D}$ projection in CAVEs is usually only optimized for the navigator. Using the [40] approach, the S3D projection is optimized for the complete audience - instead of altering the whole projection matrix, the rendered eye distance is adjusted in relation to the object of interest.

\section{Gamification}

\section{Stereoscopic Games}

Many stereoscopic games and the corresponding game consoles might be able to inspire the generation of next generation of S3D immersive systems [41]. 1983 the first S3D video games emerged on the market. The short-lived Vectrex Arcade System emerged which already provided an optional headset as viewing component that had to be used in conjunction with a CRT monitor. The headset functioned as an early version of shutter glasses. A few years later, also large companies like Nintendo entered the marked. Around 1990 a game was released on the Nintendo NES that made use of the Pulfrich effect. This review paper also describes a number of autostereoscopic approaches, early HMDs for gaming, autostereograms, as well as reflection-based approaches. It shows that the game industry always was a huge driver in innovation for $\mathrm{S} 3 \mathrm{D}$ technologies.

\section{Molecular Visualization and Simulation}

Wong et al. developed an immersive system to design molecular structure assemblies [42]. The immersive interactive system can be used to change the molecular properties of the simulated molecular membrane structure and directly observe the changes. For this purpose, the simulation environment is represented by a gaming machine-similar device in VR, which provides dials to amend the system thickness and temperature (Figure 6).

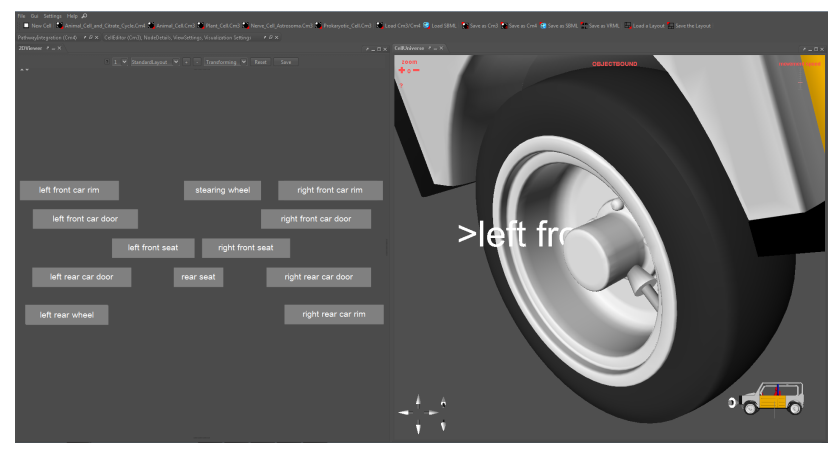

Figure 4. Hybrid-dimensional visualization and navigation: the car model can be explored in $3 D$ via the zSpace using head and a 3D stylus pen tracking (see also Figure 5 Top) as well as using the 2D map on the right side.
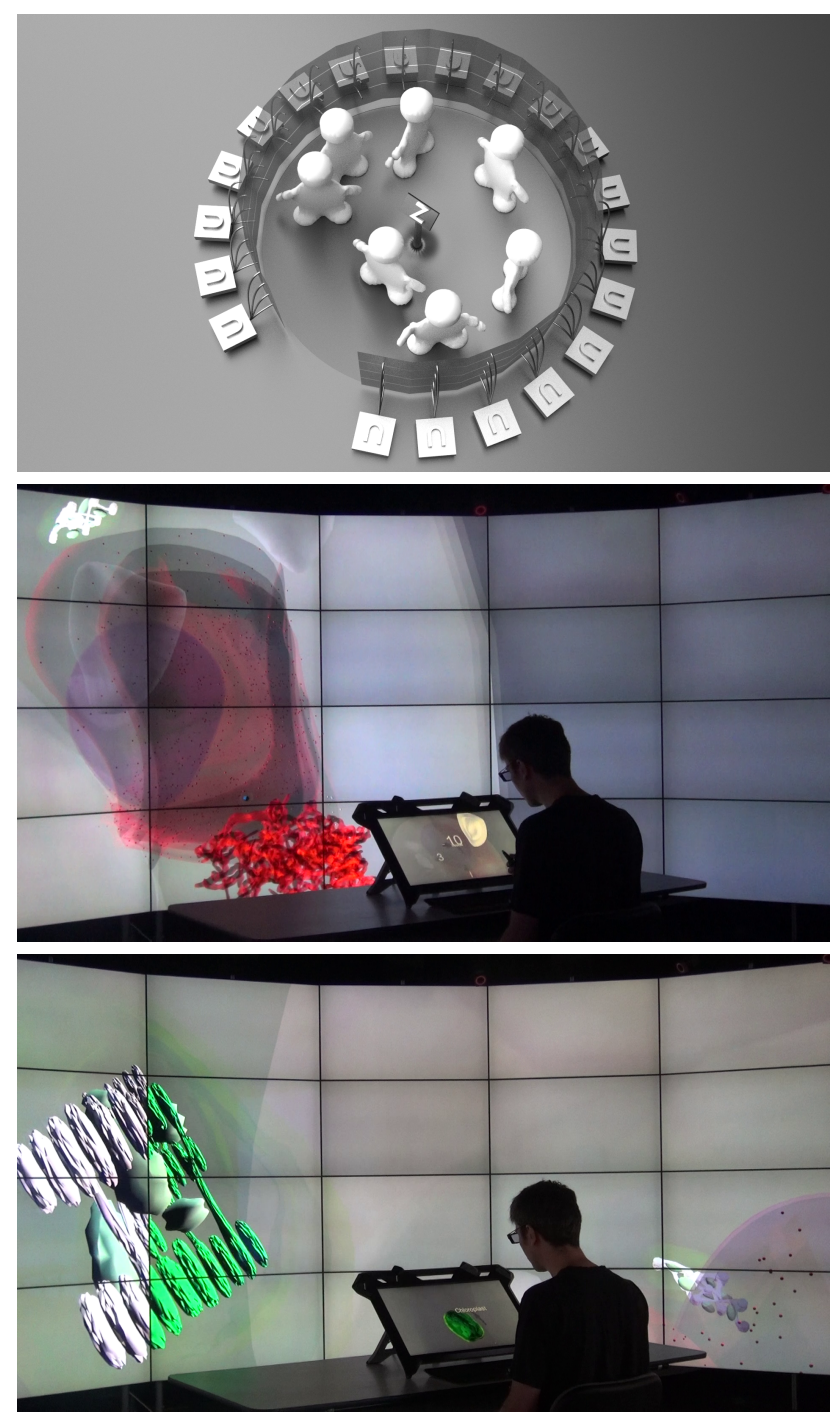

Figure 5. Stereoscopic Space Map: Top: zSpace (z) communicates with CAVE2 and enables a navigator from a central position to preview perspectives and navigate then the virtual world of the CAVE2 to this specific place. Here, 20 node computers ( $n$ ) are connected to four monitors each. Center: View from outside the cell while the navigator selects a component using the zSpace stylus pen. Bottom: Selecting the Chloroplast, the view of the CAVE2 moved inside the cell and shows the components of the Chloroplast. 


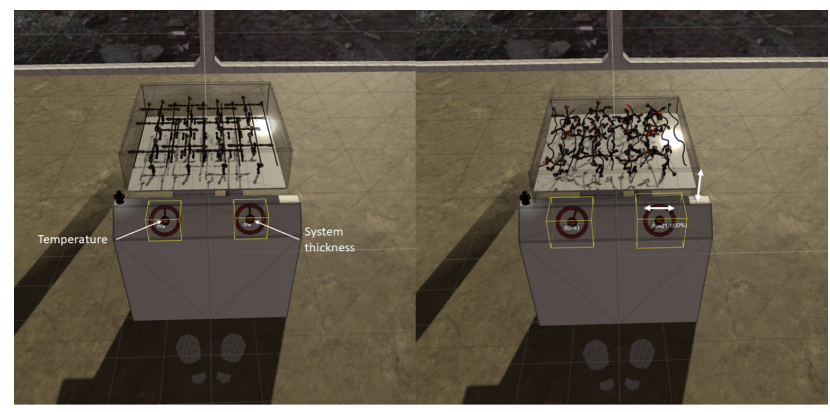

Figure 6. The biomedical simulation environment provides two dials to amend the thickness and temperature of a molecular membrane [42]. (Courtesy of (C) 2018 Hua Wong et al. via license: CC BY-NC-ND 4.0 [43], changes towards: original image was cropped by $50 \%$ ).

\section{Training Environments \\ Firefighter Training in VR}

Puel et al. designed a system called PRESTO-FF to build a realistic firefighter training environment to teach situation awareness and coordination of teams based [44]. The first prototype is based on the commercial XVR simulation system [45]. The introduced system includes a modeling facility for behaviours of people/AI on the scene according to their roles (like firefighter, victim, informer), a task-specific 3D environment (e.g. a flat), and a visual scripting system that impacts the simulation based on player's actions. In addition, a trainer is able to manipulate the system in real-time. 15 domain experts positively evaluated the system, despite low visual quality in this first prototype. A review of firefighting environments is provided by Engelbrecht et al. [46].

\section{Evacuation Training in AR}

Sharma et al. designed a Unity-based AR application helping people to evacuate from a building affected by smoke and fire [47]. A study was conducted emulating an evacuation situation. Using smart phones and tablets, the study participants were provided with a 3D map of the environment - including fire sources - and the direction towards the nearest exit were indicated. The evaluation built on 30 responses clearly showed that participants preferred this approach over traditional 2D evacuation plans. Integrated signs helped to improve orientation and participants expected this approach to be much better in real-time evacuations.

\section{Optimization of the Visual Experience}

\section{Stereoscopic Movie Evaluation}

Vatolin et al. developed a number of methods to assess the quality of stereoscopic movies and created reports of a number of popular 3D movies [48]. In this work, 10 metrics are introduced, including - besides well-known S3D issues like extreme horizontaldisparity and vertical parallax - color mismatch between views, field-of-view/scale mismatch, as well as rotation mismatch. Vatolin et al. conducted a number of reports assessing the quality S3D movies by using these metrics and more. The analysis of 105 S3D movies was concluded with the statement that in the period between 2010-2016 the S3D movie-production pipelines have considerably improved.

\section{Stereoscopic Design with Blender}

Biere et al. discussed how to design a 3D model and create a 3D-stereoscopic movie of a Chlamydomonas $r$. cell using the Open Source tool Blender [49]. The challenge here was to combine different information sources, such as $2 \mathrm{D}$, spatial, light- and electron-microscopic images as well as from publication-related data. In this way, an interpretative visualization approach had to be used. In addition, different scales had to bridged and the stereoscopic presentation had to be optimized for this purpose. This approach might be interesting wherever structural data has to be visualized that is inaccessible to the bare human eye as well as to optics, and different visual information sources have to be combined.

\section{Interaction}

\section{Hand interaction in $V R$}

MacAllister et al. presented one of the first proof of concept systems integrating the Leap Motion and Oculus Rift DK2 into a commercial engineering visualization and analysis package called Siemens' TeamcenterßLifecycle Visualization Mockup. During a study it was shown that these two devices together can provide real time, fluid interaction in a commercial engineering platform [50]. Different gestures were used for this purpose: direction-based rotation was triggered by a swipe gesture, the zoom in/out feature by a moving forward/backward gesture, and gesture tracking was turned on by closing the right fist and turned off by using the left one. Based on Fikkert et al. these mirrored gestures are highly intuitive for on/off actions [51].

\section{A Virtual User-friendly Keyboard}

A relevant problem in virtual environment is the keyboard interaction, which is normally limited to a navigation device coming with the used HMD and an ordinary keyboard which can only be used if the HMD is taken aside or in a blind manner. A virtual keyboard alternative was, e.g., developed by Jimenez et al. [52]. It is used in combination with an Oculus Rift DK2 and Leap Motion. The smart phone-based Swype algorithm is emulated in their implementation to enable a user-friendly typing experience. Also, a gaze mode was implemented that can be used for interaction purposes.

\section{Presence and Blended Spaces Presence in the Virtual Workplace}

Presence - or the "sense of feeling there" - is an important factor when designing virtual worlds and underlying user interactions. An extensive survey on Presence and related concepts was introduced by Skarbez et al. [53]. In the future it can be expected that designers have to work for extended periods of time in VR environments. Therefore, feeling comfortable and immersed into the virtual workspace will be of high relevance. Parola et al. proposed as an alternative interpretation of might be the "sense of feeling real" [54]. An important aspect of Presence is that implemented designs should take individual user differences into account which are usually reflected in the real world by individual Gestaltung of a working place. Parola et al. further investigated the "sense of feeling real" analysing a virtual climbing experience based on the variables 1) expertise in real-world activities, 2) the interaction ability, and 3) the virtual hand ownership illusion. Positive correlations between the different variables were found [55]. 


\section{Blended Spaces, Embodied Information Behavior and Mixed Reality}

West et al. discussed in the context of different VR and AR technologies embodied information behavior [56]. For this purpose, they reviewed a number of technologies and associated narratives, e.g. by analyzing advertisements. The authors observed that the proposed seamless interaction of material reality and digital data envisions: 1) immersion on-demand, 2) immersion on-the-go, 3) sensorial augmentation beyond that which is humanly possible, and 4) hybrid virtual experiences consisting of "being there" and "being here." Moreover, the authors were referencing to Benyon's "Blended Spaces" which incorporate work in blended theory to develop user experiences within and across digital-physical spaces [57]. Benyon advocates design with awareness on the ontology, topology and volatility to create correspondences and adequate associated transitions [56]. He defines presence as "the experience, that [designers] are trying to create for people" [57] and envisions a sense of presence that is multidimensional, distributed as well as transitioning between the digital and physical world.

\section{Special Environments: Underwater and in Space $A$ digital VR planetarium}

The work of Dias et al. [58] presents Galactica, a digital planetarium that proposes the use of stereoscopy to depict photorealistic scenes. The high-resolution multi-display projection system is based on OpenSceneGraph and is able to visualize astrophysical data and phenomena. More than $100 \mathrm{~K}$ stars were visualized via billboards plus more than $100 \mathrm{~K}$ additional polygons. Often, for this kind of visualizations, dome projections are used to enable a $180^{\circ}$ view of the night sky. This kind of environments is highly interesting for space R\&D.

\section{Stereoscopic Underwater Imagery}

Stereoscopic photography and filming have a long tradition and there are many different approaches available. However, special environments require highly specific solutions. The underwater project of Woods et al. had the main task to image and reconstruct the wrecks of the HMAS Sydney II and HSK Kormoran, including their debris fields using photogrammetry [59]. For this purpose, special underwater cameras were developed by the Centre for Marine Science and Technology The produced S3D footage was shown at 3D cinemas as well as at Power Walls in the HIVE of Curtin University.

\section{Data Analytics}

\section{Evaluation of Stereoscopic Rendering of Multi-} dimensional Data Visualization

Etemadpour et al. analyzed the user performance when carrying out an interactive visual exploration of multidimensional data in a CAVE [60]. For this purpose, a user study was conducted where users had to analyze the spatial data relationships of 3D scatter plots as well as cluster visualizations in the form of enclosing surfaces or hulls. The participants had to perform a set of typical analysis tasks in the CAVE and on a standard $2 \mathrm{D}$ screen. The study showed that the immersive environment improved performance for local analysis tasks, but not so much for global ones. In addition, surface-based visual encodings seemed to profit more from the VR environment than point-based renderings. However, it has to be mentioned that display fidelity and the quality of interactions are important performance-related factors.

\section{Collaborative Immersive Data Analytics}

As analysis is a crucial part in the design process, there are a number of aspects which are related to Immersive Analytics that can be applied to Immersive Design Engineering as well. As Immersive Analytics approaches were already evaluated from a number of perspectives, we can utilize related scientific observations [61, 29, 62].

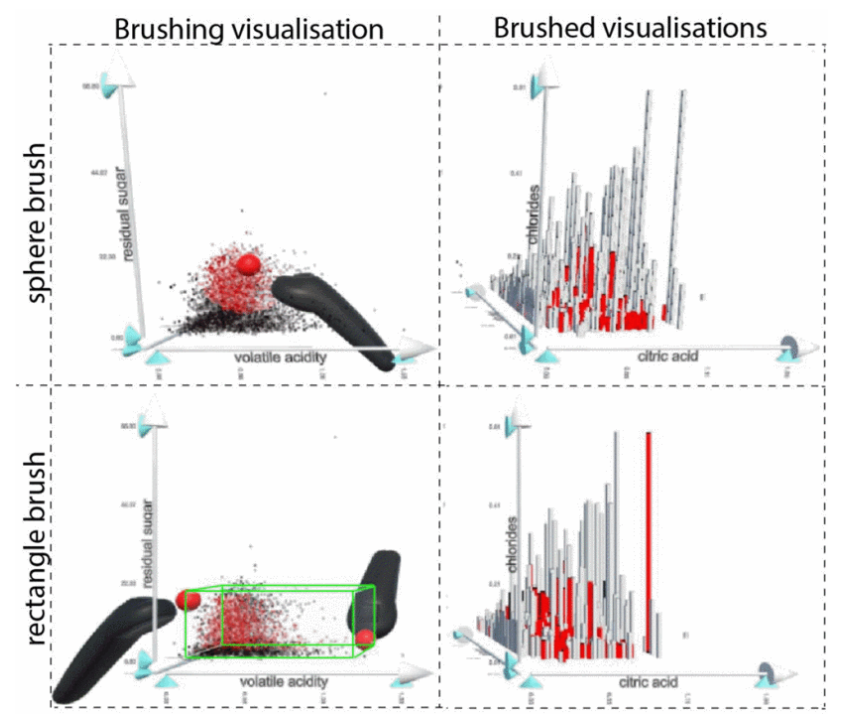

Figure 7. Abstract data visualization in virtual environments. IATK is used here to visualize multi-dimensional data. Left the scatter plots are shown, on the right the selected bar charts are highlighted in red. The selection is performed via Brushing and Linking: on the top left via a sphere selection, on the bottom left via a selection box. (Courtesy of (C)2019 Maxime Cordeil. All Rights Reserved.)

A very interesting example in this context is IATK - the Immersive Analytics Toolkit - which provides interactive authoring and exploration of data visualization in immersive environments [63]. A grammar of graphics is provided to the user which can be configured by using a GUI in Unity as well as a specific API supporting the creation of novel immersive visualization designs and interactions. Figure 7 shows how Linking and Brushing is implemented here in the virtual environment. IATK supports a number of visual designs, such as spheres, plots, quads with different sizes and colors, as well as parallel rendering of millions of items. Also, a number of VR devices were already used in this context, e.g., the Oculus Rift CV1, Meta 2 as well as the HoloLens. Approaches like this will be relevant in the future to merge VR engineering applications directly with statistical analyzes in a fluent and user-friendly fashion. Another important factor for the development of the VR work place of the future is supporting collaborations. For this purpose, IATK was extended by an early prototype called FIESTA, a free roaming Collaborative Immersive Analytics (CIA) System [64]. In contrast to many existing CIA prototypes, FIESTA allows users to collaboratively work together wherever and however they wish, untethered from mandatory physical display devices. Virtual wall can be used to 
facilitate group discussion and presentation by placing, e.g., scatter plots on those walls. For this purpose, a "tear-out" metaphor is used to duplicate visualizations from panels and freely position them afterwards in the virtual space.

\section{Multi-perspective Trajectory Visualization and Analysis}

A number of virtual display environments were discussed in the context of different research projects. Often, either CAVEs or HMDs were used. Klein et al. used an alternative approach for visualizing flight trajectory data of birds by using a multiple S3D screen setup [65]. Two screens each were placed on three wheeled monitor mounts which also carry the connected computer. In this way, the monitor setup can easily be transported to different places and configured in different ways. Figure 8 shows the "Tiled Stereoscopic 3D Display Wall" showing multiple perspectives based on bird flight data [66]. It supports stereoscopic visualization using S3D TVs. In this way, first person perspectives can be shown in parallel to overview maps in a collaborative display environment. The underlying software system TEAMwISE is based on the Cesium framework and allows multiple synchronized 3D views [67]. As the visualized data is based on GPS sensors, this kind of immersive visualization system might be highly interesting for Immersive Analytics of flight data from drones, airplanes, etc.

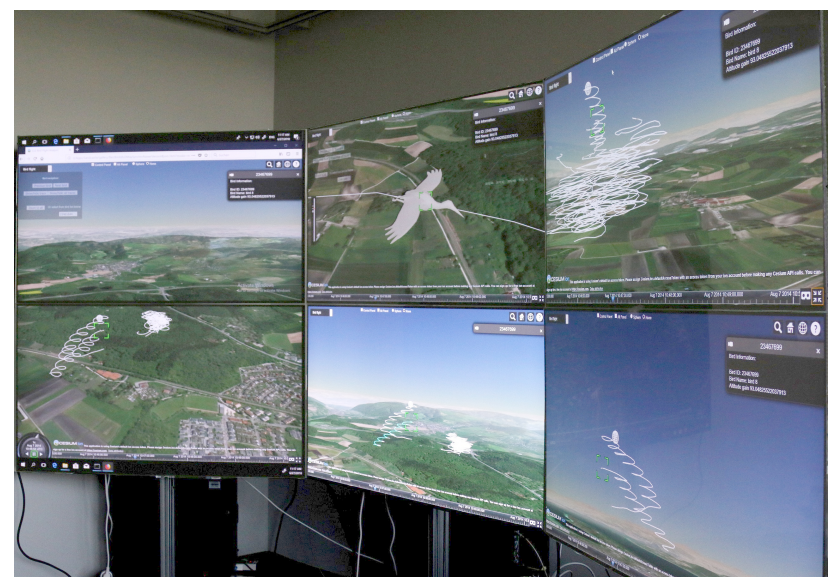

Figure 8. The Tiled Stereoscopic 3D Display Wall showing different perspectives of a bird flock trajectory data set.

\section{Commercial Projects}

\section{Virtual Studios enabling distant Group Dynamics and} Fluent Discussions

Skype, Zoom and Discord are all very well known video communication tools whose popularity drastically accelerated - Zoom in particular - during the COVID-19 lockdown in 2020. Although these are great tools for lectures, they lack dynamic interaction, floating group building, serendipity and spatial localization. Here, Gather.town - started in early 2020 by an independent group of engineers called the Siempre Collective - provides an alternative by combining an adventure gaming-like $2 \mathrm{D}$ interface with video communication [68]. By using an editor, different virtual 2D environments can be created based on simple background images, configured and extended by virtual posters, embedded Google documents, games, video streams, as well as portals to additional
Gather maps. Figure 9 shows the first prototype of the virtual Innovation Design Engineering studio at the Royal College of Art during a first test. The studio was 3D modeled based on original plans of the building and then rendered with Blender. However, Gather.town is a 2D application, but provides a good level of immersion by combining video chat with free movement on the map. This kind of environment might very well be a near-future version of virtual offices, discussion rooms, fluid presentation spaces in the context of Design Engineering.

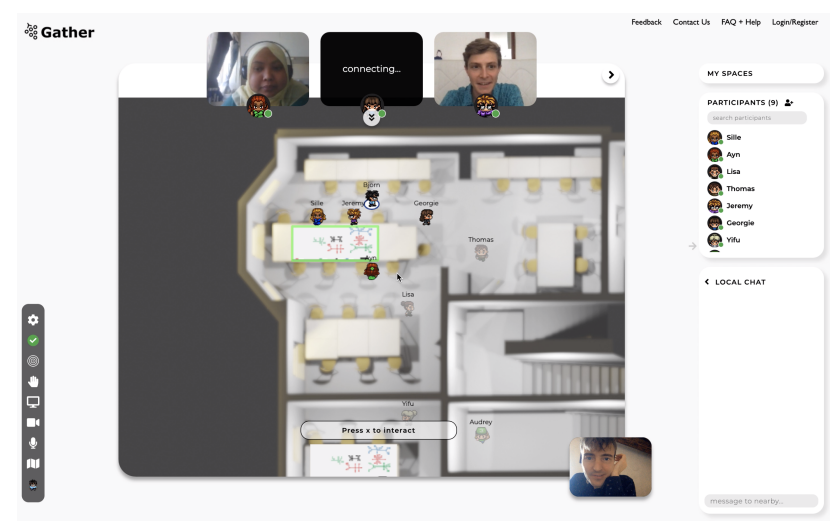

Figure 9. Gather.town showing the first prototype of the virtual IDE studio at the Royal College of Art during a test session. Students and tutors are communicating via chat and video, while moving along a 2D map.
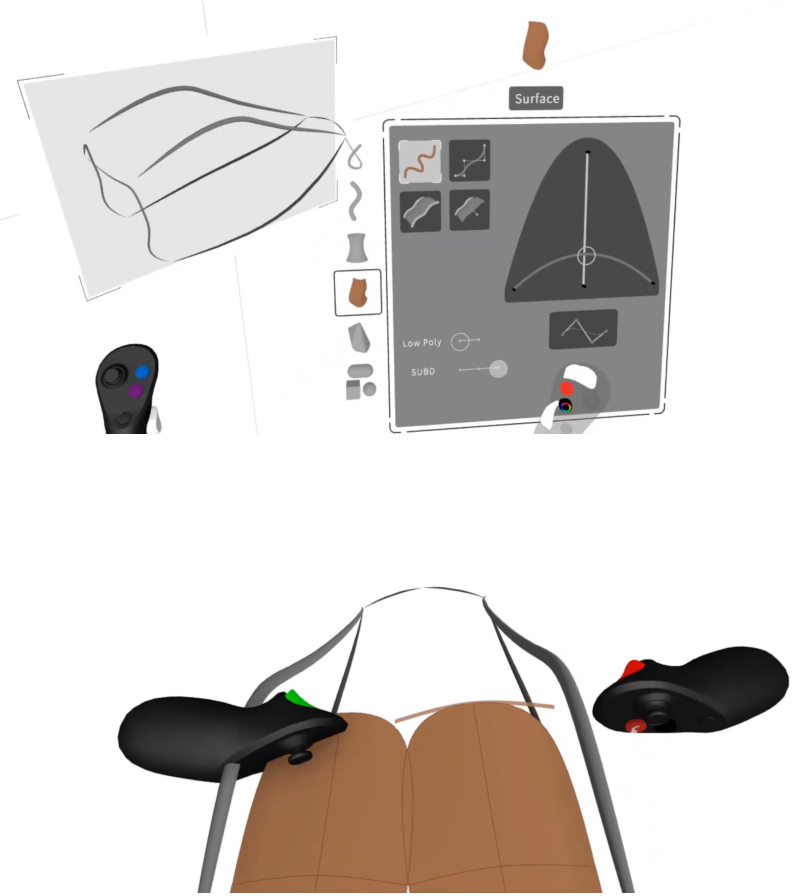

Figure 10. Gravity Sketch enables $3 D$ sketching using VR technologies for professional purposes, such as car and shoe design. Top: context menu to enable symmetric drawing with initial $3 D$ sketch in the background; the settings window can be freely positioned in the virtual space by using the controllers. Bottom: drawing a symmetric subdivision surface along the $3 D$ sketch [69]. (Courtesy of (C)2020 Gravity Sketch Ltd. All Rights Reserved.) 


\section{Freely Designing and Sketching in VR}

Gravity sketch is a project whose roots go back to the Innovation Design Engineering program (see below). The basic idea of Gravity Sketch is to provide a new way of interaction inside virtual environments by enabling 3D drawing and 3D sketching [70]. In this way, it is a drastically extended version of Google Tilt Brush, which enables painting and brushing in 3D environments with a focus on artists [71]. Gravity Sketch enables the drawing of smooth curves freehand, extruding surfaces into the third dimension and then manipulating these surfaces by grabbing and moving splines' points. Gravity Sketch can be used as a cloudbased solution to access design content directly from within the VR headset and to collaborate between different design engineers. Potential uses are car and shoe design, concept art, product design.

\section{From Student Project to Commercial AR Product - an Example}

Augmented reality currently suffers from a lack of high-quality input devices. The available state-of-the-art products have limitations when in real-world use. For example, the hand tracking used to control HoloLens 2 requires the user to keep their hands raised in front of the headset. Voice is suited to selecting categorical data but unfit for scrolling or scaling objects while handheld controllers are restrictive because they require one hand to be free for the controller.

Litho is a wearable controller for augmented reality that aims to address the aforementioned problems. The Litho controller is worn between your first and second finger. It has a capacitive trackpad on the underside, an array of motion sensors, and provides haptic feedback. Litho's design means you can wear it while doing everyday activities (e.g typing, driving, drilling).

Litho's tracking software outputs the approximate position and rotation of the controller relative to the phone or headset's camera - allowing developers to create apps that let you interact with objects in the real world, simply by pointing. This is achieved by taking data from the camera and the IMU to estimate the controller's absolute position in space. Crucially, this does not rely on external tracking systems to work, enabling use in a much wider range of scenarios (outdoors, in large areas, etc).

The Litho device, in combination with the included Software Development Kit (SDK) and suite of example projects, provides a complete package for developers looking to produce mobile AR apps featuring precise and intuitive manipulations in $3 \mathrm{D}$ space [72].

The first prototype of the controller was developed in the Innovation Design Engineering program of the Royal College of Art and Imperial College by Nat Martin. This is a two-year Master's Program. Towards the end of the first year, the students work for several weeks on their own single project: the Solo Major module (Solo Exploration from 2020). In this short amount of time, the students have the opportunity to follow the whole product design process, starting from ideation, formulating an innovation brief, research, prototyping and validation.

Figure 11 Top shows an early sketch of Nat's original idea during the ideation phase. He quickly developed a number of different ideas and started then to create an early prototype, as seen in Figure 11 Top Center. The shape of the navigation ring was $3 \mathrm{D}$ printed and the electronics were fitted into the device. For
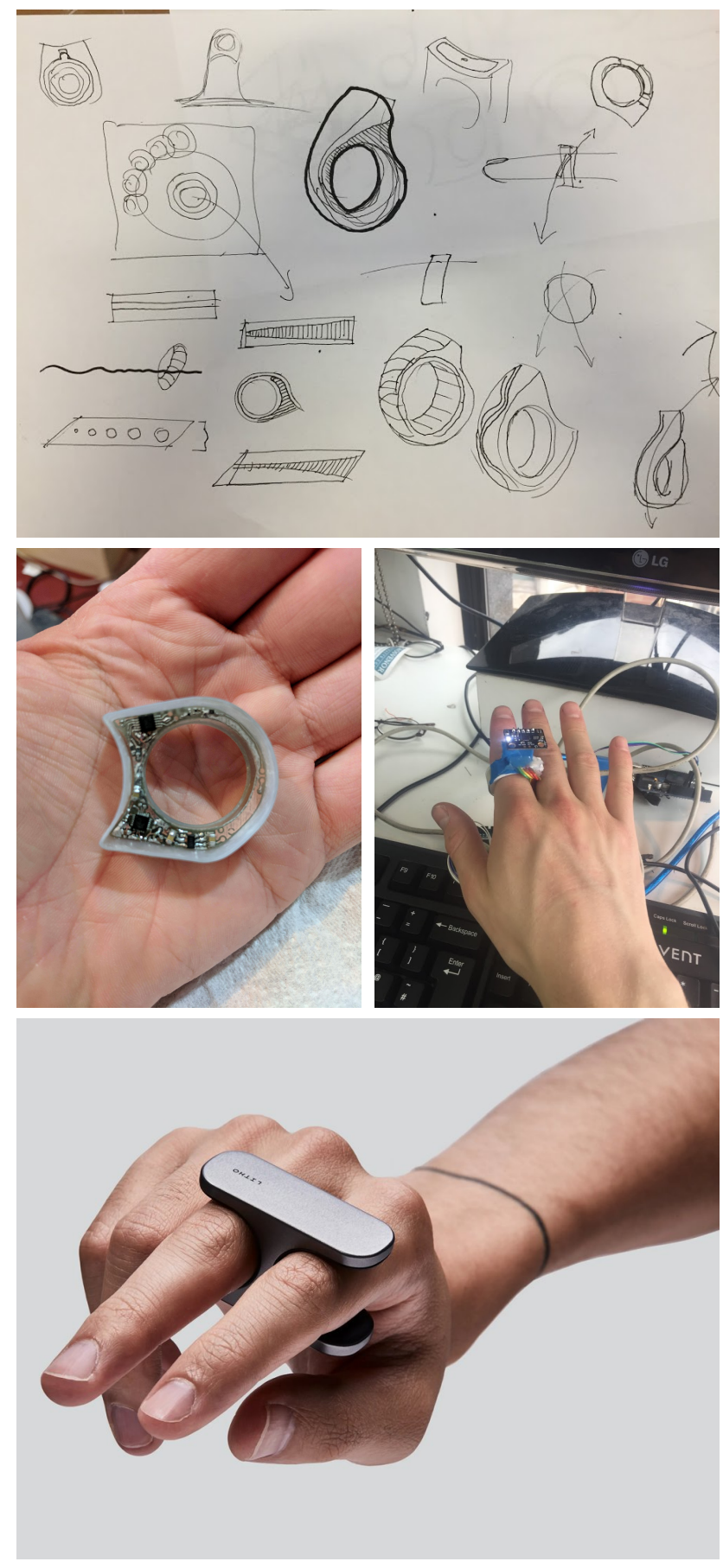

Figure 11. LITHO: Top: First sketches by Nat Martin 2017. Center: An early LITHO prototype. Bottom: The final product in 2020. (Courtesy of (C)2017-20 Nat Martin. All Rights Reserved.)

each iteration, the prototype was tested on his classmates. Even at this early stage the prototypes provided a simple 3D navigation solution. The original process video shows the idea to use this device for an augmented wayfinding application [73].

The final outputs from the project were a working prototype of the hardware and a future-facing concept video. These outputs were presented at the Royal College of Art Degree Show 
(2017). While on display at the show, Nat's project was picked up by several media outlets. This press coverage resulted in him being contacted by a number of Angel investors and Venture Capital firms. At this point, with the guidance of the two universities involved, Nat established a company to continue the development of the prototype. And four months after this initial contact from investors, they closed their first round of investment. This money enabled them to build a small team, protect their IP and mass produce and ship the product.

Based on this early working prototype, the final product was developed, as shown in Figure 11 Top Bottom. As of July 2020 Litho has been used in a wide array of fields including manufacturing, human-car interaction, education, space exploration, architecture, retail, healthcare and financial services. More information can be found at https://www.litho.cc

\section{Intuitive Surgical}

Intuitive Surgical provides S3D medical solutions for surgeries with the Da Vinci system, shown at Figure 12 [74]. On one hand, this system enables perceiving spatial distances when operators perform surgeries, on the other hand distant operation is possible. In this way, medical experts can perform or accompany highly specific surgeries from distant locations. While this device is obviously optimized for the medical sector, derivative approaches might be used in the future as part of the design engineering process at small scales where precision matters.

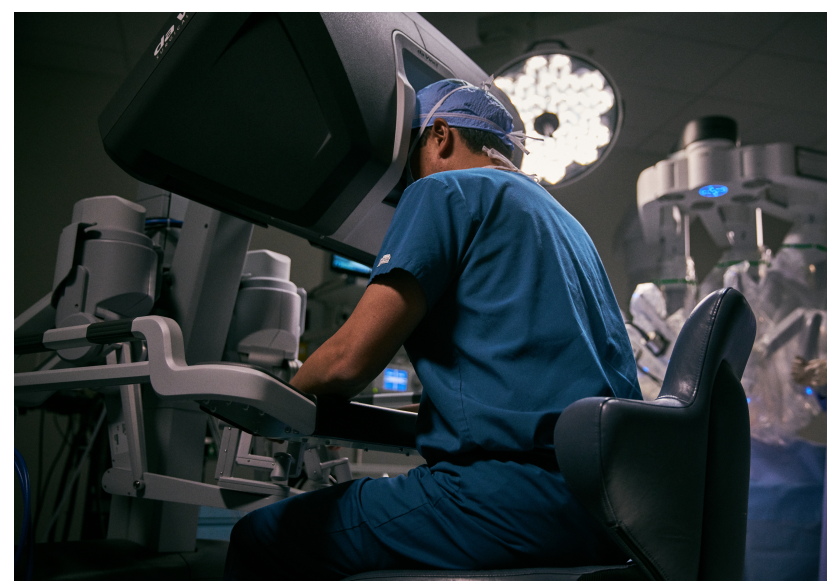

Figure 12. Da Vinci $X$ surgical system from Intuitive Surgical enabling stereoscopic distant surgeries. (Courtesy of (C)2020 Intuitive Surgical, Inc. All Rights Reserved.)

\section{Discussion and Conclusions}

In the previous chapter, we discussed a number of Immersive Design Engineering projects, or those which are related to this area and can be used as an inspiration. The projects were categorized into 10 categories:

- Virtual Cities, Landscapes and Cultural Heritage

- Vehicle Simulation and Remote Control

- Navigation

- Gamification

- Training Environments

- Optimization of Visual Experience

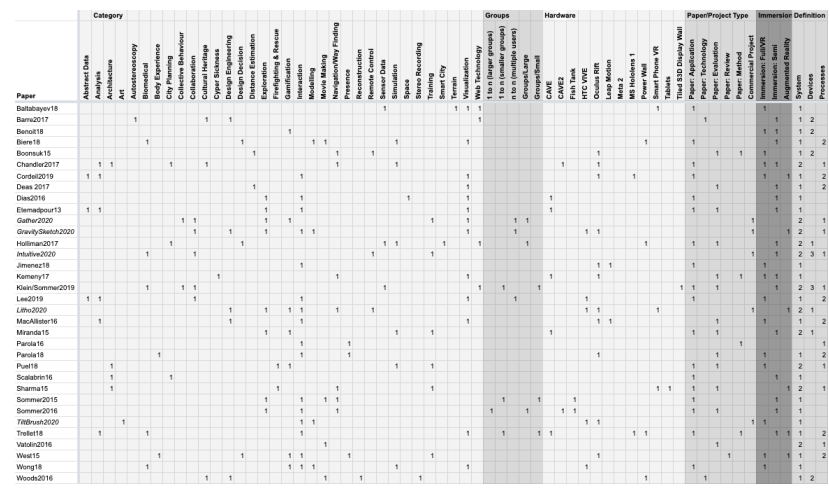

Figure 13. This is a snapshot of the comparison table listing all Immersive Design Engineering and -related projects discussed in this short review: http: //ide2020. immersive-analytics.org.

- Interaction

- Special Environments: Underwater and Space

- Data Analytics

- Commercial Projects

Of course, this differentiation is very general and the boundaries are floating. Therefore, this paper is accompanied by a comparison matrix (Figure 13) of the different tools which can be found here. It differentiates between different general categories similar to the one used in the previous chapter, as well as between different hardware, paper/project type, groups and grades of immersion. Moreover, it assigns one or more categories of the initial definition - Systems, Devices and Processes - to each of the projects.

http://ide2020.immersive-analytics.org

Based on the projects discussed, we identified a number of research questions in the context of Immersive Design Engineering projects. This non-exhaustive list will have to be extended in the future:

1. How is it possible to combine simulation, such as crowd and environment simulation, in a way that it supports the Design Engineering process?

2. How can we improve the usage of web technology in combination with immersive devices?

3. Distance estimation and spatial perception are crucial for many DE processes. It was shown that immersive technologies support the distance estimation towards close objects (0-5ft). Is there a way to improve the distance estimation in terms of far-distance objects (10-100ft).

4. How can we use immersive technologies in terms of interactive and participatory city planning in DE?

5. How can we integrate and visualize sensor data - such as temperature or air pressure - into immersive DE applications?

6. How can we integrate and utilize user's sensor data - such as galvanic skin resistance or breathing - in immersive DE applications with the purpose to improve the user experience?

7. What is the best way to integrate cloud-based data into IDE applications and how to access the data afterwards?

8. A general problem of immersive solutions: how can we improve the stereoscopic vision and perception and optimize it 
for individuals - which even might be part of a larger audience? (view-centered S3D optimization)

9. How can we optimize a virtual scene or environment to provide an optimal configuration in terms of S3D visualization (scene-centered S3D optimization)?

10. How can we merge hybrid-dimensional (i.e. 2D and 3D) visualization in immersive environments in a user-friendly way?

11. What is the best approach to merge different immersive technologies, such as zSpace/CAVE, or 3D monitor and HMD?

12. How do we optimize IDE in terms of different user scenarios. In our review, different scenarios were observed: 1) single user, 2) multiple users with the same role, usually during collaboration, 3) 1-to-n users for small groups, as well as for large groups.

13. Which lessons can we learn in the context of IDE from immersive games and the corresponding consoles? What is the impact of playfullness for IDE?

14. How to avoid cybersickness over a long period of time while working in IDE projects?

15. How to provide data analytics inside an IDE environment (directly related to Immersive Analytics)?

16. What is the best way to present data from multiple perspectives and and enable immersive comparison?

\section{Outlook}

Finally, we would like to introduce a number of new - partly disruptive ideas - which might trigger future development in the context of Immersive Design Engineering.

\section{Disruptive Display Design}

Since much of how we establish meaning in the real world is qualitative rather than quantitative, there are opportunities within Human-Computer Interaction (HCI) for qualitative displays and interfaces in relation to information presentation - especially when quantification has become a default mode or approach for information display and presentation. Qualitative displays and interfaces present information primarily through qualities of things [75]. It enables people to explore new forms of understanding information through qualities of phenomena (e.g., people, objects and events around us etc.). Figure 14 shows an example of how a qualitative interface may work in the context of a person experiencing weight or force. Here, the shape of a liquid, pressure, expansion, and the constant change of scale provides an experiential value of weight or force beyond merely providing a numerical value on a scale. These qualitative representations may improve people's understanding and experience about the relationships between meanings and phenomena. Since the possibilities of qualitative display in design and HCI has not been fully discussed in relation to virtual environments, exploring the idea of qualitative display in the context of immersive visualization could push the boundary of future interface/interaction.

\section{Multisensory System towards Synaesthesia}

Most systems discussed here strongly focus on the visual sense. Of course, HCI plays also a pivotal role in most applications. Most VR headsets nowadays are delivered with 3D interaction devices - such as the VIVE (Cosmos) controllers, or the $\mathrm{zS}$ -
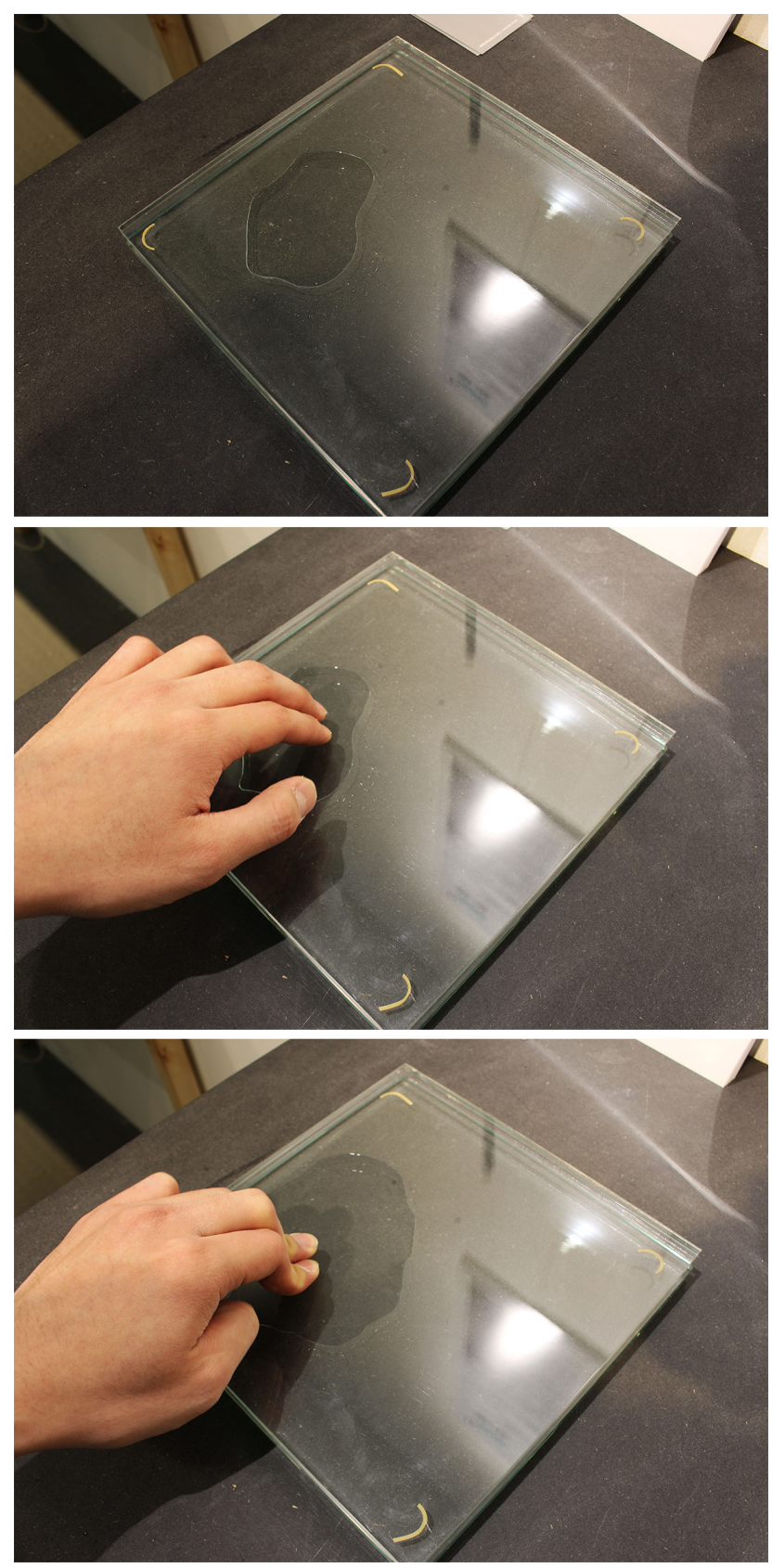

Figure 14. A non-numerical weighing scale. Liquid trapped under glass changes shape, size and scale.

pace stylus. Like the previously-mentioned devices, many provide haptics, usually by using vibration feedback. Hand tracking also plays an important role - especially in the early days of the Oculus Rift (Development Kit 2) the Leap Motion was a widelydistributed device which was attached to the Rift to provide hand tracking. While various multisensory approaches are focusing to improve user experience/interactions, there are not many attempts of using synaesthesia as a source of design material to enhance immersive experiences.

Synaesthesia is a cognitive phenomenon in which stimulation of one sensory or cognitive pathway leads to automatic and 
involuntary experiences in a second sensory or cognitive pathway - for example, tasting a candy can trigger another sensation such as seeing a shape or smelling a color. Currently, there are more than 88 types of synaesthesia, and we believe exploring such a translative property of synaesthesia can be potentially useful for reinforcing immersive experiences. For instance, haptic value can be mapped with visual value to maximize users experience and sensory stimulation. Synaesthesia and its translative property $[76$ in relation to immersive interactions are a less developed area. Exploring this area of study may push the boundary of designing immersive environments.

\section{Cognitive Interaction}

Exploring cognitive interaction beyond our sensory systems may also reinforce immersive experiences. One of our pilot studies [77] tentatively suggests that it may be possible to shape user experiences without any direct physical, sensory, and social interactions. Figure 15 shows an example of how an experience can be shaped without any direct interactions. If there is a way to generate a type of experience without any interactions, where and how can we apply this approach or phenomenon in the context of immersive interaction? While many experiences can be achieved through various types of physical, sensory and social interactions, we believe such a focus can also shape our behaviors and experiences within immersive environments.

\section{Acceleration by Immersion?}

MacAlister et al. mentioned in their work that "product design in today's globalized world requires quick turnarounds to maintain profitability" [50]. As mentioned earlier, Design Engineering is an interdisciplinary field, and therefore, the teams consist of diverse interdisciplinary backgrounds enabling concurrent design. And these teams are supposed to generate products with great speed and accuracy [78]. A challenge of the future will be to optimize the use of immersive technologies in the context of collaboration and effectiveness. Especially in the era of COVID19, distant collaboration support is essential. Effective work tools, such as visualization or product life-cycle management software can bridge gaps between diverse working backgrounds and distant work environments [79, 80,50$]$.

\section{Immersion $\neq$ Virtual Reality}

Although the term 'Immersion' is nowadays strongly associated with VR technologies, we would like to emphasize that immersion must be also seen in a broader context (we mentioned earlier a number of works discussing immersion from various perspectives). Human-centered design has been a leading paradigm in design for the past few years. However, now that the paradigm is shifting - as we can observe that too much focus on the human neglects the environment with negative implications on the own population - new approaches are required.

So how does immersive design relates to human-centered design? Immersive design takes into account different senses of the user. In the context of VR, full immersion is usually supported by an HMD, providing a $360^{\circ}$ virtual experience, whereas semiimmersion is supported by 3D fishtanks, offering a window into the virtual world while maintaining the visual access to the real world. However, if we would address this more strictly, full immersion would also address all human senses. In the context of
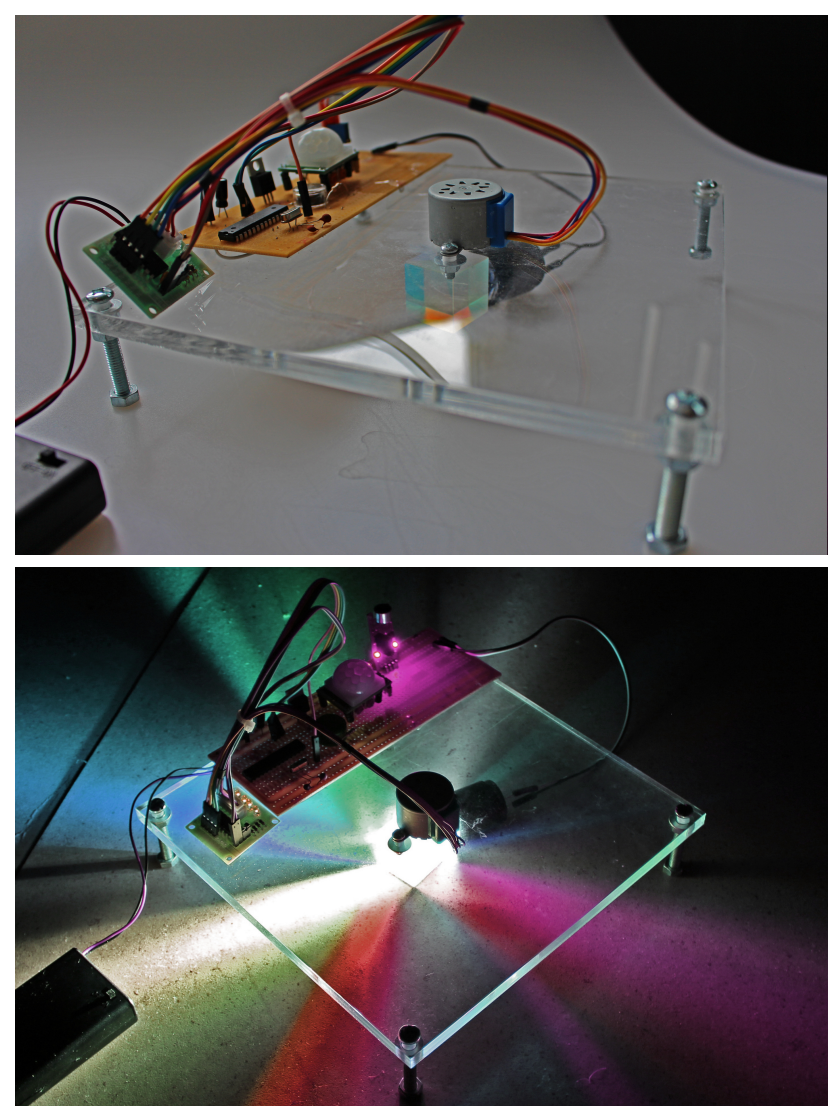

Figure 15. This unusual machine or experiment is a stationary device (left) that appears to do nothing. However, when there are no humans in its environment - detected by no sound, no motion and no light - it secretly starts to emit beams and rays of stunning colors/lights. Although it practically provides no interaction - since it will never function in front of people participants were able to experience certain playful aspects.

design and engineering, we would expect those senses to at least be supported and required to finish a particular task. A 3D modeling task of a car will require an immersive visualization, touch interaction with feedback. In an extended context, this scenario would require the support of audio (in case the exhaustion system is modeled) and olfactory senses (in case the UX of the interior is modeled). So, all these aspects are providing an immersive scenario, and in this way a very user-centered scenario. But as immersion takes different senses into account, we can argue that the simulation or emulation of the environment can also be improved - enabling a more context-aware (towards post-human-centered) design. Whereas we are building the environment around the user in the center, in this case, the degree of isolation of the user would be relatively low as he can interact with his environment using several senses and increasing awareness.

In contrast to many other immersive visualization approaches that are often more of theoretical nature or for technique evaluation purposes, the product of design engineering is usually a concrete artefact which emerges from the virtual world into the real world. Therefore, the immersive experience produces a spatial model, like in our example, a car. This bilaterally increases the value of the immersive approach. 
However, as shown by the initial research agenda extracted from this review: there are many questions in this field to be more precisely elaborated in the near future.

\section{References}

[1] M. A. Robinson, P. R. Sparrow, C. Clegg, K. Birdi, Design engineering competencies: future requirements and predicted changes in the forthcoming decade, Design Studies 26(2), pp. 123-153 (2005).

[2] N. Greffard, F. Picarougne, and P. Kuntz, Visual community detection: An evaluation of 2D, 3D perspective and 3D stereoscopic displays, International Symposium on Graph Drawing, pp. 215-225 (2011).

[3] J. P. McIntire, P. R. Havig, and E. E. Geiselman. What is 3D good for? A review of human performance on stereoscopic 3D displays, Head- and Helmet-Mounted Displays XVII, 8383, pp. 83830X-1-13 (2012).

[4] M. A. Robinson. How design engineers spend their time: Job content and task satisfaction. Design studies 33(4), 391-425 (2012).

[5] International Data Group (IDC), AR and VR Headsets Will See Shipments Decline in the Near Term Due to COVID-19, But Long-term Outlook Is Positive, According to IDC, https: //web.archive.org/web/20200531133955/https: //www.idc.com/getdoc.jsp?containerId=prUS46143720 (last accessed on 2020-06-17).

[6] C. Cruz-Neira, D. J. Sandin, T. A. DeFanti, R. V. Kenyon, and J. C. Hart, The CAVE: audio visual experience automatic virtual environment, Communications of the ACM, 35(6), pp. 64-72 (1992).

[7] A. Febretti, A. Nishimoto, T. Thigpen, J. Talandis, L. Long, J., D. Pirtle, T. Peterka, A. Verlo, M. Brown, and D. Plepys, CAVE2: a hybrid reality environment for immersive simulation and information analysis., in IS\&T/SPIE Electronic Imaging, pp. 864903-864903-12 (2013).

[8] 3D TV is dead - The Independent (2017), https://www.independent.co.uk/life-style/gadgets-and-tech/news/3dtv-dead-last-manufacturers-lg-sony-give-up-tech-trend-glasseshome-cinema-television-a7546091.html (last accessed on 2020-0809).

[9] Oculus - VR Headsets \& Equipment, https://www.oculus .com (last accessed on 2020-06-25).

[10] HTC VIVE ${ }^{\mathrm{TM}}$ - Discover Virtual Reality Beyond Imagination, https://www.vive.com (last accessed on 2020-06-25).

[11] Varjo XR-1 - The world's most advanced virtual and mixed reality devices, https://varjo.com. (last accessed on 2020-06-25).

[12] zSpace - AR/VR Learning Experiences, https://zspace.com (last accessed on 2020-06-25).

[13] 3D Pluraview - Beam Splitter Technology, Passive 3D-stereo display, https://www.3d-pluraview.com (last accessed on 202006-25).

[14] Looking Glass Factory - The World's Leading Holographic Display, https://lookingglassfactory.com (last accessed on 2020-0625).

[15] Alioscopy - Glasses-free 3D displays, http://www.alioscopy. com (last accessed on 2020-06-25).

[16] Microsoft HoloLens - Mixed Reality Technology for Business, https://www.microsoft.com/en-us/hololens (last accessed on 2020-06-26).

[17] Mechdyne - Audiovisual Consulting Services \& AV/IT Design Solutions, https://www .mechdyne.com (last accessed on 2020-0625).
[18] Vicon - - Award Winning Motion Capture Systems, https:// Www. vicon.com/ (last accessed on 2020-06-30).

[19] D. Checa, A. Bustillo, A review of immersive virtual reality serious games to enhance learning and training, Multimedia Tools and Applications, 79(9), pp. 5501-5527 (2020).

[20] Unity Real-Time Development Platform - 3D, 2D VR and AR Engine, https://unity3d.com (last accessed 2020-08-09).

[21] Unreal Engine - The most powerful real-time 3D creation platform, https://www . unrealengine. com/ (last accessed 2020-08-09).

[22] WorldViz - Virtual Reality for Training and Research, https:// www. worldviz.com (last accessed on 2020-06-25).

[23] OGRE - Open Source 3D Graphics Engine - Home of a marvelous rendering engine, https://www.ogre3d.org (last accessed 202008-09).

[24] McGraw-Hill Dictionary of Scientific and Technical Terms, 6th ed., McGraw-Hill Education (2003).

[25] W. Lidwell, K. Holden, J. Butler, Universal principles of design, revised and updated: 125 ways to enhance usability, influence perception, increase appeal, make better design decisions, and teach through design. Rockport Pubublishers (2010).

[26] M. Csikszentmihalyi, Flow: The Psychology of Optimal Experience, Harper Collins Publishers (1991).

[27] F. Hülsmann, N. Mattar, J. Fröhlich, I. Wachsmuth. Simulating wind and warmth in virtual reality: conception, realization and evaluation for a cave environment, JVRB-Journal of Virtual Reality and Broadcasting 11(10) (2014).

[28] R. De La Barré, R. Bartmann, M. Kuhlmey, B. Duckstein, S. Jurk, S. Renault, A new design and image processing algorithm for lenticular lens displays. Electronic Imaging 2017(5), pp. 194-199 (2017).

[29] B. Sommer, D. Barnes, S. Boyd, T. Chandler, M. Cordeil, K. Klein, T. D. Nguyen, H. Nim, K. Stephens, D. Vohl, S. Wang, E. Wilson, J. McCormack, K. Mariott, F. Schreiber, 3D-Stereoscopic Immersive Analytics Projects at Monash University and University of Konstanz, IS\&T Electronic Imaging: Stereoscopic Displays and Applications XXVIII Proceedings, IS\&T, Springfield, VA (2017).

[30] T. Chandler, B. McKee, E. Wilson, M. Yeates, M. Polkinghorne. A New Model of Angkor Wat: Simulated Reconstruction as a Methodology for Analysis and Public Engagement. Australian and New Zealand Journal of Art 17(2), pp. 182-194 (2017).

[31] M. Scalabrin, L. A. Ripamonti, D. Maggiorini, D. Gadia. Stereoscopy-based procedural generation of virtual environments. Electronic Imaging 2016(5), pp. 1-7 (2016).

[32] N. Holliman, M. Turner, S. Dowsland, R. Cloete, T. Picton, Designing a Cloud-based 3D Visualization Engine for Smart Cities, Electronic Imaging 2017(5), pp. 173-178 (2017).

[33] A. Baltabayev, A. Gluschkow, J. Blank, G. Birkhölzer, M. Kern, F. Klopfer, L.-M. Mayer et al., Virtual Reality for Sensor Data Visualization and Analysis, Electronic Imaging 2018(3), pp. 451-1 (2018).

[34] M. R. Miranda, H. Costa, L. Oliveira, T. Bernardes, C. Aguiar, C. Miosso, A. B. S. Oliveira, A. C. G. C. Diniz, D. M. G. Domingues, Development of simulation interfaces for evaluation task with the use of physiological data and virtual reality applied to a vehicle simulator, The Engineering Reality of Virtual Reality 2015, p. 939207 (2015).

[35] W. Boonsuk, Usability of stereoscopic view in teleoperation, In Stereoscopic Displays and Applications XXVI, pp. 93911 G (2015).

[36] L. M. Deas, R. S. Allison, B. Hartle, E. L. Irving, M. Glaholt, L. M. Wilcox, Estimation of altitude in stereoscopic-3D versus 2D real-world scenes, Electronic Imaging 2017(5), pp. 41-47 (2017).

[37] M. Trellet, N. Férey, J. Flotyński, M. Baaden, P. Bourdot, Semantics 
for an integrative and immersive pipeline combining visualization and analysis of molecular data, Journal of integrative bioinformatics 15(2) (2018).

[38] A. Kemeny, P. George, F. Mérienne, F. Colombet, New VR navigation techniques to reduce cybersickness, Electronic Imaging 2017(3), pp. 48-53 (2017).

[39] B. Sommer, S. J. Wang, L. Xu, M. Chen, F. Schreiber. Hybriddimensional visualization and interaction-integrating $2 \mathrm{D}$ and $3 \mathrm{D}$ visualization with semi-immersive navigation techniques Autoren, IEEE Big Data Visual Analytics (BDVA), pp. 1-8 (2015).

[40] B. Sommer, A. Hamacher, O. Kaluza, T. Czauderna, M. Klapperstück, N. Biere, M. Civico, B. Thomas, D. G. Barnes, and F. Schreiber. Stereoscopic Space Map - Semi-immersive Configuration of 3D-stereoscopic Tours in Multi-display Environments. Electronic Imaging, Proceedings of Stereoscopic Displays and Applications XXVII, 2016(5), pp. 1-9 (2016).

[41] I. Benoit, E. Kurland. The History of Stereoscopic Video Games for the Consumer Electronic Market. Electronic Imaging 2018(4), pp. 290-1 (2018).

[42] H. Wong, J. Prévoteau-Jonquet, S. Baud, M. Dauchez, N. Belloy, Mesoscopic Rigid Body Modelling of the Extracellular Matrix SelfAssembly, Journal of integrative bioinformatics 15(2) (2018).

[43] Creative Commons License: Attribution-NonCommercialNoDerivatives 4.0 International (CC BY-NC-ND 4.0), https://creativecommons.org/licenses/by-nc-nd/4.0/ (last accessed on 2020-08-10).

[44] D. Puel, An authoring system for VR-based firefighting commanders training, Electronic Imaging 2018(3), pp. 469-1 (2018).

[45] XVR Simulation - Incident command training tool for safety and security, https://www.xvrsim.com. (last accessed on 2020-08-05).

[46] H. Engelbrecht, E. Lindeman, S. Hoermann, S, A SWOT analysis of the field of virtual reality for firefighter training, Frontiers in Robotics and $\mathrm{AI}(6)$, p. 101 (2019).

[47] S. Sharma, S. Jerripothula, An indoor augmented reality mobile application for simulation of building evacuation, The Engineering Reality of Virtual Reality 2015, p. 939208 (2015).

[48] D. Vatolin, A. Bokov, M. Erofeev, V. Napadovsky. Trends in S3Dmovie quality evaluated on 105 films using 10 metrics, Electronic Imaging 2016(5), pp. 1-10 (2016).

[49] N. Biere, M. Ghaffar, A. Doebbe, D. Jäger, N. Rothe, B. M. Friedrich, R. Hofestädt, F. Schreiber, O. Kruse, B. Sommer, Heuristic modeling and 3D stereoscopic visualization of a Chlamydomonas reinhardtii cell, Journal of integrative bioinformatics 15(2) (2018).

[50] A. MacAllister, T.-P. Yeh, E. Winer, Implementing Native Support for Oculus and Leap Motion in a Commercial Engineering Visualization and Analysis Platform.” Electronic Imaging 2016(4), pp. 1-11 (2016).

[51] W. Fikkert, P. Van Der Vet, G. van der Veer, A. Nijholt, Gestures for large display control, International Gesture Workshop, pp. 245-256 (2009).

[52] J. G. Jimenez, J. P. Schulze, Continuous-Motion Text Input in Virtual Reality, Electronic Imaging 2018(3), pp. 450-1 (2018).

[53] R. Skarbez, F. P. Brooks Jr, M. C. Whitton, A survey of presence and related concepts, ACM Computing Surveys 50(6), pp. 1-39 (2017).

[54] M. J. Parola, S. Johnson, R. West, Turning presence inside-out: Metanarratives, Electronic Imaging 2016(4), pp. 1-9 (2016).

[55] M. J. Parola, R. West, R. Herrington, C. Adams, M. Beyer, B. Davis, K. Hays et al., From Being There To Feeling Real: The Effect Of
Real World Expertise On Presence In Virtual Environments, Electronic Imaging 2018(3), pp. 434-1 (2018).

[56] R. West, M. J. Parola, A. R. Jaycen, C. P. Lueg, Embodied information behavior, mixed reality and big data, The Engineering Reality of Virtual Reality 2015, pp. 93920E (2015).

[57] D. Benyon, Presence in blended spaces, Interacting with Computers 24(4), pp. 219-226 (2012).

[58] M.S. Dias, J. d'Alpuim, P. Caetano, Galactica, a Digital Planetarium for Immersive Virtual Reality Settings, International Journal of Creative Interfaces and Computer Graphics (IJCICG), 7(1), pp. 19-39 (2016).

[59] A. Woods, P. Helmholz, A. Bickers, J. Hollick, An underwater odyssey: the mission to image the wrecks, From Great Depths The Wrecks of HMAS Sydney II and HSK Kormoran, pp. 255-277 (2016).

[60] R. Etemadpour, E. Monson, L. Linsen, The effect of stereoscopic immersive environments on projection-based multi-dimensional data visualization. 17th IEEE International Conference on Information Visualisation, pp. 389-397 (2013).

[61] T. Chandler, M. Cordeil, T. Czauderna, T. Dwyer, J. Glowacki, C. Goncu, M. Klapperstück, K. Klein, K. Marriott, F. Schreiber, E. Wilson, Immersive Analytics, Big Data Visual Analytics, BDVA 2015, pp. 73-80 (2015).

[62] B. Sommer, M. Baaden, M. Krone, A. Woods, From virtual reality to immersive analytics in bioinformatics, Journal of integrative bioinformatics 15(2) (2018).

[63] M. Cordeil, A. Cunningham, B. Bach, C. Hurter, B. H. Thomas, K. Marriott, T. Dwyer, Iatk: An immersive analytics toolkit, IEEE Conference on Virtual Reality and 3D User Interfaces (VR) 2019, pp. 200-209 (2019).

[64] B. Lee, M. Cordeil, A. Prouzeau, T. Dwyer, FIESTA: A Free Roaming Collaborative Immersive Analytics System, ACM International Conference on Interactive Surfaces and Spaces 2019, pp. 335-338 (2019).

[65] K. Klein, B. Sommer, H. T. Nim, A. Flack, K. Safi, M. Nagy, S. P. Feyer, Y. Zhang, K. Rehberg, A. Gluschkow, M. Quetting, W. Fiedler, M. Wikelski, F. Schreiber, Fly with the flock: immersive solutions for animal movement visualization and analytics, Journal of the Royal Society Interface, 16(153), 20180794 (2019).

[66] B. Sommer, A. Diehl, M. Aichem, P. Meschenmoser, K. Rehberg, D. Weber, D., Y. Zhang, K. Klein, D. Keim, F. Schreiber, Tiled Stereoscopic 3D Display Wall-Concept - Applications and Evaluation. Electronic Imaging, 2019(3), pp. 641-1-641-15 (2019).

[67] Cesium - Changing how the world views 3D, https://www. cesium.com (last accessed on 2020-08-06).

[68] Gather - Virtual spaces for conferences, https://gather.town/ conferences/ (last accessed on 2020-08-08).

[69] Gravity Sketch - New Feature 4 - SubD Snapping, https://www. youtube .com/watch?v=EznyDjGY36c (last accessed on 2020-08$09)$.

[70] Gravity Sketch - Think in 3D. Create in 3D, https://www. gravitysketch.com (last accessed on 2020-06-25).

[71] Tilt Brush by Google, https://www.tiltbrush.com (last accessed on 2020-08-09).

[72] LITHO - A Controller for the real world, https://www.litho.cc (last accessed on 2020-06-25).

[73] LITHO Process Video - an Augmented Reality Ring Controller (2017), https://www youtube. com/watch?v=qNUJE_VNYdY (last accessed on 2020-08-08). 
[74] Intuitive Surgical - Robotic-Assisted Surgery, https://www. intuitive.com (last accessed on 2020-06-25).

[75] D. Lockton, D. Ricketts, S. Aditya Chowdhury, C. H. Lee. Exploring qualitative displays and interfaces, $\mathrm{CHI}$ Conference Extended Abstracts on Human Factors in Computing Systems 2017, pp. 18441852 (2017).

[76] C. H. Lee, D. Lockton, J. Stevens, S. J. Wang, S. Ahn, SynaestheticTranslation Tool: Synaesthesia as an Interactive Material for Ideation, Extended Abstracts of CHI Conference on Human Factors in Computing Systems 2019, pp. 1-6 (2019).

[77] C. H. Lee, D. Lockton, J.E. Kim, Exploring Cognitive Playfulness Through Zero Interactions, ACM SIGCHI Conference on Designing Interactive Systems 2018 (DIS 2018), pp. 63-67 (2018).

[78] M.L. Swink, J.C. Sandvig, V .A. Mabert, Customizing concurrent engineering processes: Five case studies, Journal of Product Innovation Management 13(3), pp. 229-244 (1996).

[79] W. Shen, Q. Hao, W. Li, Computer Supported Collaborative Design: Retrospective and Perspective, Computers in Industry 59(9), pp. 855862 (2008).

[80] F. Belkadi, E. Bonjour, M. Camargo, N. Troussier, B. Eynard, A situation model to support awareness in collaborative design, International Journal of Human-Computer Studies 71(1) pp. 110-129 (2013). 


\section{JOIN US AT THE NEXT EI!}

IS\&T International Symposium on

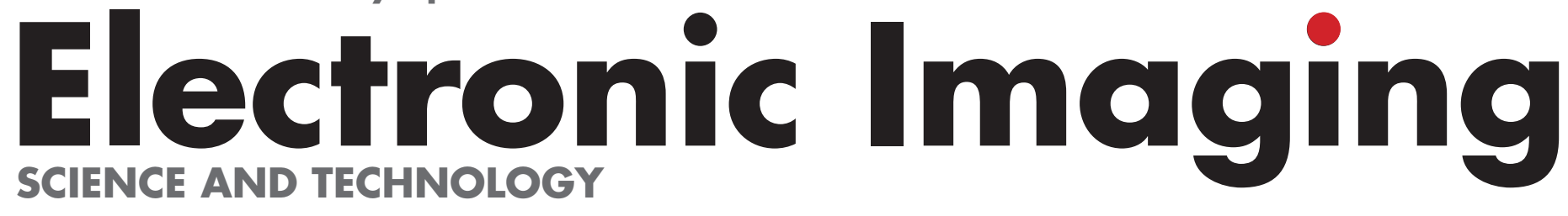
Imaging across applications ... Where industry and academia meet!
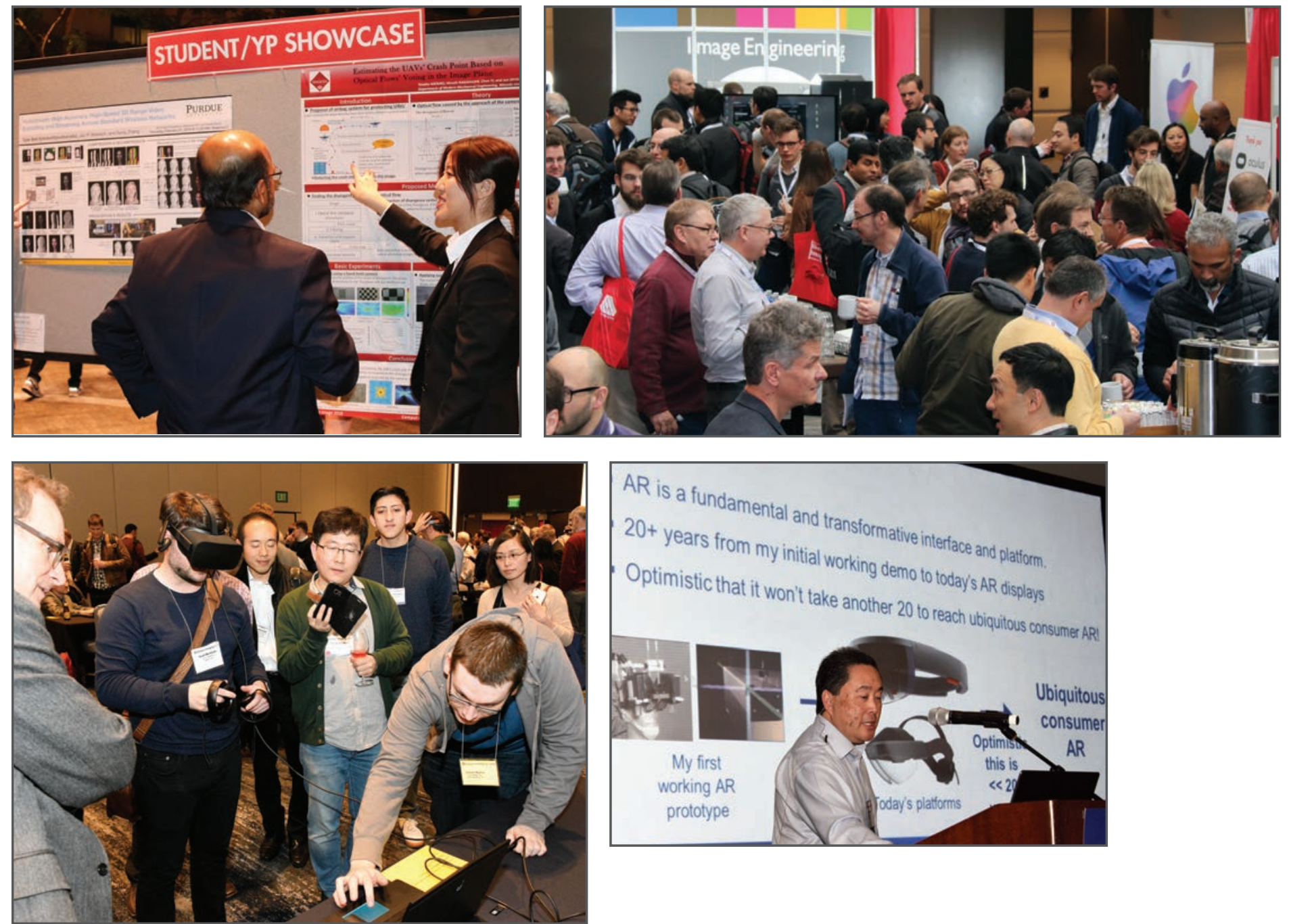

- SHORT COURSES • EXHIBITS • DEMONSTRATION SESSION • PLENARY TALKS • - INTERACTIVE PAPER SESSION • SPECIAL EVENTS • TECHNICAL SESSIONS •

www.electronicimaging.org

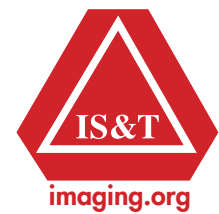

